\title{
REVIEW ON TANTALUM EXTRACTION BY DIFFERENT METHOD IN KENTICHA TANTALUM MINE, ETHIOPIA
}

\author{
Elias Kassahun Woldeselsassie \\ School of Resource and Environmental Engineering \\ Wuhan University of Technology \\ Wuhan, Hubei, China
}

\begin{abstract}
In Ethiopia, a huge and potentially minable tantalum mineral reserve is available and extracted by open-pit mining. The separation process of tantalum in kenticha mine site is gravity separation because of the density difference among the associated mineral or the gangue.

Niobium and tantalum are chemically similar and are related to one another in nature which makes it hard to isolate. Basically, the most usable technology for separation of tantalum from the ore is gravity separation, which is the old and economical method. This method is radically changed by the chemical or solvent extraction method and flotation method. This strategy is fundamentally changed by the substance or dissolvable extraction technique and buoyancy technique.

This paper reviews the overall processing system of kenticha tantalum mining and gives some clue on advancement and other recent technology used by other countries.
\end{abstract}

Key Word: Tantalum, Niobium, Gravity Separation

\section{INTRODUCTION}

Tantalum plays a significant role in the nation's mining industry, which contributes fundamentally to the country's GDP. Ethiopia is invested with a novel mineral store that places the nation among the best ten producers of tantalum on the world.

The nation is underlain by an alternate kind of rocks (Metamorphic, Sedimentary, and Igneous) running in age from Precambrian to recent. The geological evolution gave rise to a wide range of mineral resources (base, precious and rare metals) and rocks for various uses (gemstones, dimension stone and industrial, construction and etc.)

\author{
Dereje Demissie, Tamrat Mekonnen \\ School of Resource and Environmental Engineering \\ Wuhan University of Technology \\ Wuhan, Hubei, China
}

Mineral investigation exercises are very constrained and not coordinated to distinguish potential economic deposits.

Similarly, there are indications of petroleum resources and coal deposits in the sedimentary basins that are not yet very well studied.

The supply of mineral raw materials will form the basis for the development of new manufacturing industries (ex. Fertilizers, cement, ceramic, etc.), that would play a critical role in the earning of foreign exchange, increase the levels of import substitution, particularly for manufactured commodities, and create employment opportunities in the primary and secondary industries.

At present the tantalum focus is sold on the global market, however metallurgical endeavor is expected to expel up to $0.5 \%$ uranium from the concentrate and to include an incentive by delivering tantalum mixes of tantalum metal.

Niobium is a rare, soft, grey and ductile transition metal with the symbol $\mathrm{Nb}$. Niobium is used in superconducting magnets, memorial coins, therapeutic gadgets, adornments, curve cylinder seals, capacitors, optical focal point, indicator, atomic applications, superconducting RF cavities.

Tantalum was found by Anders Gustaf Ekeberg (17671813), in 1802. It is rare metal hard, blue-dark, and glistening progress metal, with the image Ta. Tantalum is used in composites and wires, careful instruments, response vessels and funnels, ultra-highrecurrence electron tubes, focal point, vacuum heater parts, watches, and tantalum being synthetically idle is additionally utilized in capacitors as a platinum substitute. Columbite, tantalite, columbite-tantalite (Colton), pyrochlore, and euxenite constitute the major primary sources for niobium and tantalum. 
Tantalum and niobium mineral deposits are broad over the globe, yet a considerable lot of these deposits are low grade in both niobium and tantalum content.
Brazil and Australia have the largest high-grade niobium and tantalum resource; thus, they are the significant world producer of these metals pursued by Canada, Mozambique, and Ethiopia.

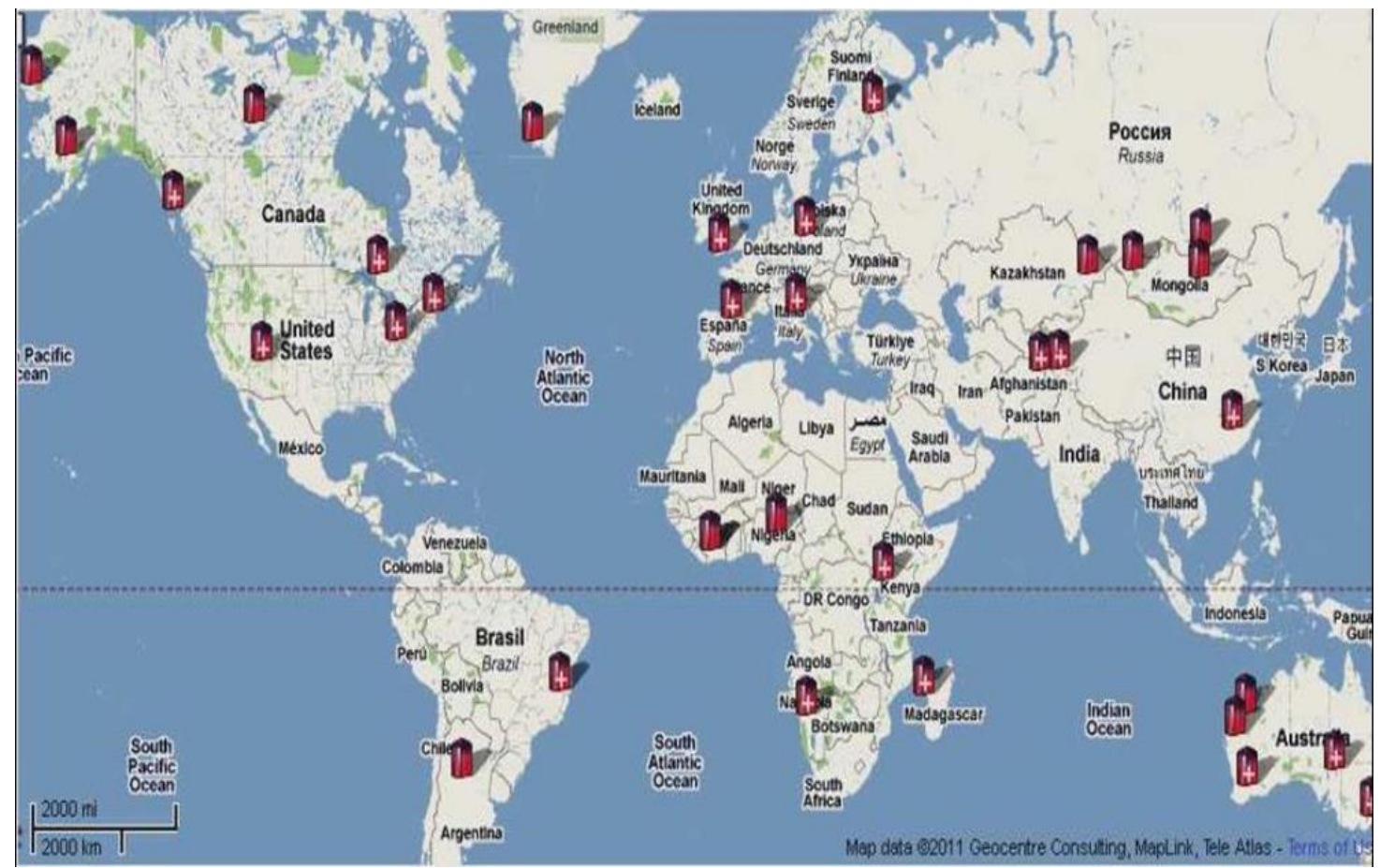

Figure 1 the world map showing selected areas (symbol) of Coltan distribution.

\section{PLANT DESIGN BASE DATA OF THE MINE SITE}

\subsection{Capacity of Ore Dressing Plant}

- Raw ore processing capacity, a year: 1070000 t/y, (Scrubber capacity; 75 $\mathrm{t} / \mathrm{h} * 2 \mathrm{pcs})$

\subsection{Property of Row Material}

- Ore type: weathering type ore of pegmatite

- Valuable minerals: columbite, tantalite

- Gangue minerals: quartz, feldspar, mica, clay soil and so on

- Particle size of raw ore: $125 \mathrm{~mm}$ over $-5 \%$

- Volume weight of raw ore: $1.7 \mathrm{t} / \mathrm{m} 3$

- Specific gravity of raw ore: $2.6 \mathrm{t} / \mathrm{m} 3$

- Moisture of raw ore: $5 \%$

- Concentrate content of raw ore: $200 \mathrm{~g} / \mathrm{t}$

- Specific weight of concentrate: $3.5 \sim 4.5$ $\mathrm{t} / \mathrm{m} 3$

- $\quad \mathrm{Ta} / \mathrm{Nb}$ ratio from 1:1 to $3: 1$

\subsection{Working System}

- Working days, a year: $350 \mathrm{~d} / \mathrm{y}$

- Working times, a day: $20.4 \mathrm{~h} / \mathrm{d}$ (85\% of equipment operating ratio)

- Shifts: 3 shifts/day

\subsection{Ore and Product Working Condition \\ - Cut-off grade $0.01 \%$ Ta2O5 \\ - Recovery 65-70\%}

\section{PRESENT PROCESSING METHOD OF THE PLANT}

The raw ore is supplied to feed hopper by loader $(3.5 \mathrm{~m} 3)$ from storage site. The $+125 \mathrm{~mm}$ size boulders are separated by bar screen at feed hopper, and these boulders thrown away to waste site. The under size $(-125 \mathrm{~mm})$ the ore enters in trammel scrubber by pressure water from feed hopper. In trammel scrubber, ore is divided in to two products; $-16 \mathrm{~mm}$ and +16 to $-125 \mathrm{~mm}$. The $+16 \mathrm{~mm}$ to $-125 \mathrm{~mm}$ size products are thrown away by belt conveyor to the waste site; at this time, in belt conveyor visible concentrate is picked by hand and this picked concentrate is kept in concentrate store. 
The under-size product (pulp) from the scrubber $(-16 \mathrm{~mm})$ goes to fix screen site by channel. In fixed screen site, $-16 \mathrm{~mm}$ size product is fed to fix screen (44mm MOS and 380) here the oversize product $(+3$ to $-16 \mathrm{~mm})$ are fed to the jigger and the under-size product $(-3 \mathrm{~mm}$ pulp) is fed to fine classifier of dewatering. In jig site, the concentrate ( 4 to $-16 \mathrm{~mm}$ ) is picked by hand and kept in concentrate store.

The jig tailing is transported to tailing dump by channel. The undersize products from the jig fed to coarse grain main table site. From fine classifier of dewatering, pulp is divided in to two; $-0.2 \mathrm{~mm}$ size pulp (over flow) and +0.2 to $-3 \mathrm{~mm}$ size pulp (under flow), and water pressure classifier pulp is divided in to two; +0.2 to $-1 \mathrm{~mm}$ size pulp (over flow) and +2 to $-3 \mathrm{~mm}$ size pulp (under flow). These pulps with flow product of jig are fed to the main table process.

From main table process, concentrate goes to magnetic separator and tailing goes to scavenging table. The concentrate from these scavenging table process and main table goes to tailing basin. In magnetic separator, tailing (Iron) is kept independently and concentrate is fed to cleaning table (final table). The concentrate from the final table process goes to drying process (iron plate electrical drier) and tailing goes again to final scavenging table. Finally, concentrates from both final scavenging table and cleaning table goes to drying process and tailing goes to tailing basin.

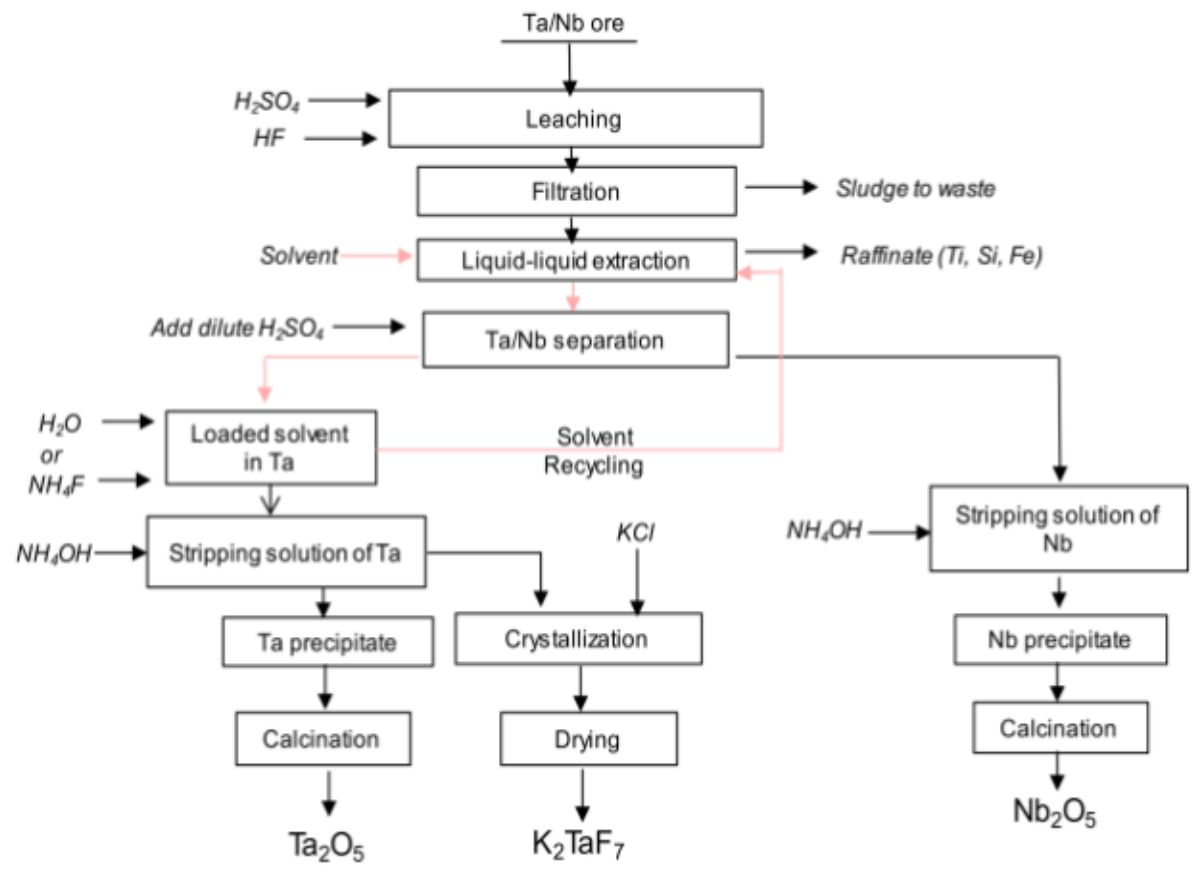

Fig 2 General gravity separation Process scheme for $\mathrm{Ta}$ and $\mathrm{Nb}$ production

\section{ALTERNATIVE EXTRACTION METHOD OF TANTALUM}

There are a number of recent technologies profoundly used by another tantalum producing countries such as liquid-liquid extraction and separation, combined model for the beneficiation of tantalum and production of fine tantalum powder by electrochemical method for tailings though we go through only two basic technologies that is liquid-liquid extraction and separation and combined model for the beneficiation of tantalum.
Separation factors of greater than one indicates that the two metals can be separated. This does not, however, give an indication of the ease of separation or the number of stages which may be required, (Ritcey and Ashbrook, 1984).

The liquid-liquid extraction has got many benefits such as very large capacities are possible with a minimum of energy consumption, when other standard separation methods fail or require expensive equipment or energy cost like kenticha power consumption, and also heat and sensitive product are produced at ambient or moderate temperature. Separation of small contents of high boiling impurities 
in aqueous solutions. In the normal thermal separation, the complete water content as to be withdrawn by a very energy intensive evaporation process. The combined technology encompasses the crushing and grinding unit, the gravity separation unit, magnetic separation unit and electrostatic separation units therefore it has many advantages comparing to other technological advancements because high rate of recovery can be achieved by this method as well as it reduces the machinery cost.

With respect to $\mathrm{Nb}$ and Ta recovery, many works have been studied - in detail - the possibilities of extracting $\mathrm{Nb}$ and $\mathrm{Ta}$ by liquid-liquid extraction method. Maiorov et al. (2001) regarding the possibility of $\mathrm{Nb}$ and Ta recovery with n-octanol from $\mathrm{HF}$ acid solutions ( $\mathrm{Nb}$ about $0.11 \mathrm{M}$ and $\mathrm{Ta}$ as low as about $0.0045 \mathrm{M}$ ) while containing large amounts of $\mathrm{Ti}(2-3 \mathrm{M})$. They recovered $95.7 \%$ and $84.1 \%$ of $\mathrm{Ta}$ and $\mathrm{Nb}$, respectively. More-over, Mayorov and Nikolaev (2002) as well as Anatoly et al. (2004) carried out the liquid-liquid extraction process of $\mathrm{Ta}-\mathrm{Nb}$, as a result of processing tantalite and columbite, by2-octanol from fluoride-sulfuric acid solutions.

In another way as M. Toure et al.,2018 mentioned that selective extraction of Tantalum and Niobium by Alkyl-Acetophenone in hydrofluoric medium, $\mathrm{Ta}^{5+}$ and $\mathrm{Nb}^{5+}$ form very stable monomeric complexes, each of them forming two types of complexes such as $\mathrm{TaF}^{72-}, \mathrm{TaF}^{6-}$ and $\mathrm{NbOF}^{52}-\mathrm{NbF}^{6-}$. The equilibrium between complexes is governed by the concentration of $\mathrm{HF}$ and elements ( $\mathrm{Ta}$ or $\mathrm{Nb}$ ), and it can be described as follows:

For the $\mathrm{Ta}: \mathrm{TaF}^{72-}+\mathrm{HF} \mathrm{TaF}^{6-}+\mathrm{HF}^{2-}$

And for the $\mathrm{Nb}$ : $\mathrm{NbOF}^{52-}+3 \mathrm{HF} \mathrm{NbF}^{6-}+\mathrm{HF}^{2-}+\mathrm{H}_{2} \mathrm{O}$.

\section{RESULT AND DISCUSSION}

\section{a. Analysis on Jigger}

As only two jigs are working properly out of five jiggers, so that there will be an overload on the jiggers consequently the jigger screen will be abraded early. Additionally, the jigger screens are older and not a default screen for the jigger i.e. when they changed the screens, they use a modified screen which do not fulfills the manufacturer manual requirement because of cost and availability of spare parts. Due to the above reasons most of the concentrate goes to the jig tailing as a result the recovery will abruptly decreased.

\section{b. Analysis on Shaking Table}

The main problems on the shaking tables are the abrasion of the riffles and the unused of inappropriate wash water requirement as an input. In addition to this the oversize of materials comes from the jig screens because of the abraded screens allows some of the oversize concentrate to the shaking tables. As a result, the riffles will be smooth and the concentrate is left through the tailing. From these the life of the shaking tables will be very short and the maintenance cost of the machine will be high. Therefore, at the end the overall recovery from the shaking tables will be decreased. The Holman Wilfley table has Wash Water requirement of 20 to $351 / \mathrm{min}$. Feed dilution to approximately $25-30 \%$ w/w solids, so that they have to follow these optimum working rate for the entire shaking table.

\section{c. Analysis on Spirals}

The streams in a spiral concentrator are controlled by three cutters or ports in the stream discharge, each of which is adjustable to control grade and recovery but they are abraded and results in a mixing of the concentrator with the tailings. As well as they sometimes do not use the spirals at all times.

\section{d. Analysis of the tailing material}

From the data obtained from the report on tailing there is a potentially recoverable fine tantalum pent oxide. Therefore, an electrochemical method in the reduction of tantalum compounds ( $\mathrm{TaCl} 5$ or $\mathrm{K} 2 \mathrm{TaF} 7$ ) in molten salt can be used to boost their economy. The production of tantalum powder through an electrochemically mediated reaction (EMR) also demonstrated the possibility of a new process that was suitable for controlling the purity and morphology of the metal powder deposit

\section{e. Analysis on the Technology for Tantalum Recovery}

There are a number of recent technologies profoundly used by another tantalum producing countries such as liquid-liquid extraction and separation, combined model for the beneficiation of tantalum and production of fine tantalum powder by electrochemical method for tailings through only two basic technologies that is liquid-liquid extraction and separation and combined model for the beneficiation of tantalum.

The liquid-liquid extraction has got many benefits such as very large capacities are possible with a minimum of energy consumption, when other standard separation methods fail or require expensive 
equipment or energy cost like kenticha power consumption, and also heat and sensitive product are produced at ambient or moderate temperature. Separation of small contents of high boiling impurities in aqueous solutions. In the normal thermal separation, the complete water content as to be withdrawn by a very energy intensive evaporation process.

The combined technology encompasses the crushing and grinding unit, the gravity separation unit, magnetic separation unit and electrostatic separation units therefore it has many advantages comparing to other technological advancements because high rate of recovery can be achieved by this method as well as it reduces the machinery cost.

\section{CONCLUSION}

Gravity separation technique is suitable with respect to cost effectiveness and environment. However, this system is very old and as we have seen in the field most of the machines are not working properly. Therefore to improve the overall recovery of the plant the jigs must be either replaced or well-maintained to work properly, for the shaking table the riffles were more or less smoothened so that they must be changed by another suitable riffles with the specification of the machines model or they must be uses an optimum amount of water as per quality of the riffle. The tailing pond is another potential to get the fine tantalum so proper investigation and frequent recycling is needed. From the tailing pond (almost half of the pond) we can get 350 tons of fine tantalum, when they recycle it there will be economical and they increases the overall recovery from the tantalum resource.

The technological advancement should be taken in to account for the future because most of the new technologies are cost effective, selective, less labor intensive except the machine operators so that they should think about this technology for the future. Generally a radical change on the present equipment should be made to improve the recovery of tantalum by the present system, the tailing which is considered as profitable should also be recycled and a suitable and adaptable technology.

\section{ACKNOWLEDGMENT}

Firstly, we would like to thanks Wuhan University of Technology to give this golden chance and my deepest gratitude is goes to Professor Yubiao Li for giving very attractive, fruitful and important lecture and research idea. My special thanks again go to my supervisor professor chi for giving special advice
VIII.

\section{REFERENCE}

1. Baba, A.A, Adeko a, F.A, and Faseki, M (2005), "A Study of The Kinetics of The Dissolution of a Nigerian Tantalite Ore in Hydrochloric Acid", Ife Journal of Science, vo .1, no 2, 221-227

2. H.C. Starck, Boyan Yuan and Toru H. Okabe "GmbH 2001 Tantalum and Niobium "the keys to the markets of the future Materials Transactions", Vol. 48, No. 10 (2007) pp. 2687 to 2694 .

3. J. A. Abols and P.M Grady Gekko 2008, "Systems Maximizing Gravity Recovery through the Application of Multiple Gravity Devices", Journal of Minerals \& Materials Characterization \& Engineering, Vol. 4, No. 2, pp 85-93, 2005.

4. Kabangu Mpinga John, 2012, Extraction and Separation of Tantalum and Niobium from Mozambican Tantalite by Solvent Extraction in The Ammonium Difluoride-Octanol System", Dissertation submitted in partial fulfillment of the requirements for the degree of MSc (Applied Science), University of Pretoria.

5. Maiorov, V.G. et al., 2001. Extractive recovery of $\mathrm{Ta}(\mathrm{V})$ and $\mathrm{Nb}(\mathrm{V})$ with octanol from HF acid solutions containing large amounts of Ti (IV). Russian Journal of Applied Chemistry 74.

6. Mayorov, V.G., Nikolaev, A.I., 2002.

Tantalum(v) and niobium(v) extraction by octanol. Hydrometallurgy 66, 77-83.

7. M. Toure, "Tantalum and Niobium Selective Extraction by Alkyl-Acetophenone" August 2018.

8. Motlalepula Nete, may 2013, separation and purification of niobium and tantalum from synthetic and natural compounds

9. Stephen R. Young, Cabot Supermetals, Bernic Lake, Manitoba, 2010, "Pushing the Limits of Gravity Separation", SGS Lakefied Research Limited, QemSCAN Anaysis of Tantalum Deportmentin MGS902 Tai ing\|, 8901-214 MI6001-Jun03.

10. Ritcey, G.M., Ashbrook, A.W., 1984. Solvent extraction principles and applications to process metallurgy: part I. Process Metallurgy, vol. 1. Elsevier, Amsterdam, p. 361.

11. El-Hussaini.O.M., El-Hakam Mahdy, M.A., 2002. Extraction of niobium and tantalum from nitrate and sulphate media by using MIBK. Miner. Process. Extract. Metall. Rev. 22 (4), 633-650.

12. Gupta, C.K., Suri, A.K., 1994. Extractive Metallurgy of Niobium. CRC Press, London. 98 $\mathrm{pp}$ 